\title{
Metamorfose Planar Via Métodos Level Set e Particle Level Set para a Reconstrução de Superfícies Tridimensionais
}

\author{
D.I. FAZANARO e H. PEDRINI* \\ Recebido em 27 janeiro, 2013 / Aceito em 19 agosto, 2013
}

\begin{abstract}
RESUMO. Inicialmente centralizadas na solução de problemas em Dinâmica dos Fluidos, as fronteiras dinâmicas, com o advento da modelagem mais robusta provida pelo método Level Set, expandiram os limites originais de sua aplicabilidade. Especificamente à área de Modelagem Geométrica, os trabalhos até então apresentados, relacionando o Level Set à reconstrução de superfícies tridimensionais, concentram-se na reconstrução a partir de uma nuvem de dados dispersos no espaço; a abordagem baseada em fatias planas paralelas e transversais ao objeto a ser reconstruído evidencia-se ainda incipiente. Este trabalho propõe-se, logo, a analisar a viabilidade do Level Set para a reconstrução tridimensional, oferecendo uma metodologia que agregue, simultaneamente, as ideias comprovadamente eficientes já publicadas sobre a aproximação em questão e as propostas para contornar as limitações inerentes ao método ainda não satisfatoriamente tratadas, em particular a suavização excessiva de características finas dos contornos em evolução sob o Level Set. Relativamente a esse ponto, o emprego da variante Particle Level Set é sugerido como uma solução, por sua intrínseca capacidade comprovada para a preservação de massa de fronteiras dinâmicas. Ao final, conjuntos de dados sintéticos e reais são utilizados para avaliar a metodologia de reconstrução de superfícies tridimensionais apresentada qualitativamente.
\end{abstract}

Palavras-chave: Level Set, Particle Level Set, metamorfose, reconstrução.

\section{INTRODUÇÃO}

Modelar a evolução de fronteiras dinâmicas sempre apresentou-se como uma tarefa desafiadora. O problema pode variar de simples fronteiras evoluindo uniformemente a curvas aleatórias mais complexas, nas quais a intensidade da velocidade que rege a evolução depende de um grande número de variáveis.

As técnicas pioneiras para solucionar essa questão manifestavam-se numericamente instáveis ou incapazes de lidar, de forma robusta, com mudanças na topologia da fronteira [9]. O método Level Set (LS), ao substituir a perspectiva paramétrica lagrangiana pela euleriana de equações

*Autor correspondente: Helio Pedrini

Instituto de Computação, Universidade Estadual de Campinas, 13083-852 Campinas, SP, Brasil.

E-mails: helio.pedrini@gmail.com; helio@ic.unicamp.br 
diferenciais parciais (EDP) [2], possibilitou a obtenção de resultados com uma acurácia significantemente aperfeiçoada.

Ao longo dos últimos anos, o método permaneceu sendo amplamente estudado pela comunidade científica. Em particular, a aplicação do Level Set à segmentação de imagens vem sendo eficiente e largamente explorada $[11,12]$, não compartilhando de igual entusiasmo a reconstrução de superfícies tridimensionais. Majoritariamente, os trabalhos de pesquisa relativos a esse tema concentram-se na reconstrução de formas a partir de um conjunto de dados dispersos no espaço, utilizando-se o Level Set na deformação de uma superfície inicial até a otimalidade.

A reconstrução tridimensional por meio de fatias planas paralelas constitui uma tradicional abordagem à reconstrução de superfícies. Entretanto, para casos em que a amostragem das fatias é, de alguma forma, falha (por exemplo, baixa taxa de amostragem e fatias adjacentes muito espaçadas entre si ou com espaçamento variável), a triangulação resultante de técnicas tradicionais pode retornar um objeto com uma superfície pouco suave ou, inclusive, divergir do teoricamente esperado.

Nilsson et al. [7] propõem uma solução para essa questão, empregando o Level Set para gerar fatias intermediárias entre as duas de cada par de fatias originais, por meio de metamorfose planar. Apesar de eficiente, o estudo não aborda, explicitamente, casos de contornos com cantos, extensões pontiagudas ou regiões de curvatura acentuada, características geométricas de uma fronteira suavizada em excesso quando evoluída sob a ação do método [8]. Por sua vez, o Particle Level Set (PLS), uma variante do original Level Set, revelou-se uma técnica robusta para a conservação de massa de fronteiras em evolução dinâmica, sendo capaz, presumidamente, de contornar o problema citado da suavização excessiva [8].

Este artigo propõe uma metodologia para reconstrução de superfícies tridimensionais por meio da técnica Particle Level Set para a metamorfose planar entre fatias distintas, cuja amostragem apresente alguma das singularidades citadas. O total de fatias, entre originais e intermediárias geradas, é empregado na reconstrução do objeto em questão.

\section{CONCEITOS TEÓRICOS}

Seja a função tridimensional $\phi$, a qual, à medida que a superfície por ela representada evolui no espaço, a evolução das curvas de nível 0 de $\phi$ delinea, implicitamente, a propagação da fronteira fechada $\Gamma$ no plano. Enuncia-se o problema de valor inicial (PVI) modelador, sob a ótica euleriana, da propagação temporal de $\Gamma$ com velocidade $F$ de sentido arbitrário como

$$
\begin{gathered}
\phi_{t}+F \cdot|\vec{\nabla} \phi|=0 \\
\text { Fronteira }=\Gamma(t)=\left\{(x, y) \in \mathbb{R}^{2} \mid \phi(x, y, t)=0\right\} \\
F \text { de sentido arbitrário, } t>0
\end{gathered}
$$

conhecidos $\Gamma(0)$ - isto é, a posição inicial da fronteira - e os modelos de $F$ e $\phi$, sendo $\phi_{t}$ a derivada temporal de $\phi$. A Equação 2.1 é denominada Equação Level Set. 
Especificamente à função tridimensional $\phi$, as funções distância sinalizada mostraram-se a escolha ideal, desde o início dos estudos sobre o Level Set $[8,9]$. Expressa pela lei

$$
\left\{\begin{array}{lll}
\phi(x, y, t)=d_{\Gamma}(x, y), & & \text { se }(x, y) \notin \Omega \cup \Gamma \\
\phi(x, y, t)=0, & & \text { se }(x, y) \in \Gamma \\
\phi(x, y, t)=-d_{\Gamma}(x, y), & & \text { se }(x, y) \in \Omega
\end{array}\right.
$$

em que $\Omega$ denota a região interna à fronteira $\Gamma$ e $d_{\Gamma}(x, y)$, a distância euclidiana de um ponto $(x, y)$ qualquer do plano ao ponto $P \in \Gamma$ mais próximo àquele, para qualquer tempo $t$ da evolução as funções distância sinalizada experimenta o elevado nível de suavidade exigido para uma evolução consistente e apurada de $\Gamma$.

No contexto do processo de reconstrução, o método Level Set é empregado na automatização da metamorfose planar, pela qual o contorno em uma das fatias originais do problema (denominada fatia origem) deforma-se continuamente, até assumir, por completo, a forma do contorno na fatia imediatamente adjacente (fatia destino).

Assim, para $\phi_{\text {origem }}$ e $\phi_{\text {destino }}$ as respectivas funções distância sinalizada das fatias origem e destino, evolui-se a metamorfose via Level Set resolvendo-se, iterativamente, o PVI

$$
\left\{\begin{array}{l}
\phi_{t}^{n+1}+\left(\phi^{n}-\phi_{\text {destino }}^{n}\right) \cdot\left|\vec{\nabla} \phi^{n}\right|=0 \\
\phi^{0}=\phi_{\text {origem }}^{0}
\end{array}\right.
$$

sendo $\phi^{n}=\phi_{\text {origem }}^{n}=\phi_{\text {origem }}(x, y, n)$ a função distância sinalizada que representa, implicitamente, a fatia intermediária gerada ao final da $n$-ésima iteração da metamorfose da fatia origem. A Equação 2.3 é resolvida para cada par de fatias originais do problema.

A essa perspectiva euleriana do método Level Set, o híbrido Particle Level Set introduz partículas pontuais lagrangianas, de modo a rastrear a posição da fronteira ao longo da resolução do problema, utilizando-as para a correção de eventuais erros causados por dissipação numérica.

No início da metamorfose de cada par de fatias, logo, as partículas são aleatoriamente sedimentadas, em uma banda ao redor da fronteira origem, e passivamente evoluídas, conforme o valor interpolado da velocidade $F=\phi^{n}-\phi_{\text {destino }}^{n}=\phi^{n}-\phi_{\text {destino }}^{0}$ para cada uma delas. A eventual localização, na região externa da fronteira, de partículas inicialmente depositadas na região interna (e vice-versa), após a evolução, sinaliza a ocorrência de erros na posição da fronteira. Tais partículas, ditas escapadas, são, então, empregadas na reconstrução das regiões da fronteira afetadas por esses erros numéricos.

\section{METODOLOGIA}

As fatias originais do problema, todas representadas por matrizes binárias, são lidas aos pares, sequencialmente. Para cada par, pré-processam-se ambas as fatias origem e destino, de modo a adaptá-las às configurações geométricas exigidas pelo processo computacional, conforme explanado nos próximos parágrafos. 
No primeiro passo do pré-processamento, as fatias são redimensionadas para dimensões quadradas, caso a altura e a largura das fatias não sejam iguais, procedimento este necessário para viabilizar o próximo passo de centralização dos contornos: se o redimensionamento não fosse implementado, regiões dos contornos, eventualmente, ultrapassariam as dimensões da fatia após a translação executada pela centralização.

Neste segundo passo, os contornos presentes nas fatias origem e destino são centralizados geometricamente em suas respectivas fatias, para que haja a sobreposição dos seus centroides. A centralização dos contornos garante não somente uma completa automatização da metamorfose entre as fatias [4], mas, principalmente, a evolução, pelo método Level Set, apenas das regiões do contorno da fatia origem que não estão perfeitamente sobrepostas a quaisquer regiões do contorno da fatia destino.

Pré-processadas as fatias origem e destino, principia-se a execução do Level Set, com a determinação das funções distância sinalizada das fatias origem e destino, a desempenharem os papéis das respectivas funções $\phi_{\text {origem }}$ e $\phi_{\text {destino }}$ ao longo de toda a futura etapa de geração das fatias intermediárias, segundo a convenção apresentada pela Equação 2.2.

Na sequência, determina-se o passo temporal $\Delta t$ da equação

$$
\frac{\phi^{n+1}-\phi^{n}}{\Delta t}+\hat{H}^{n}\left(\phi_{x}^{-}, \phi_{x}^{+} ; \phi_{y}^{-}, \phi_{y}^{+}\right)=0
$$

obtida por meio da discretização da Equação 2.3 e em que $\hat{H}^{n}\left(\phi_{x}^{-}, \phi_{x}^{+} ; \phi_{y}^{-}, \phi_{y}^{+}\right)$representa a aproximação da Hamiltoniana $H\left(\phi_{x}, \phi_{y}\right)=\left(\phi^{n}-\phi_{\text {destino }}^{n}\right) \cdot\left|\vec{\nabla} \phi^{n}\right|$ após a $n$-ésima iteração e $\phi_{x}^{-}$e $\phi_{x}^{+}\left(\phi_{y}^{-}\right.$e $\left.\phi_{y}^{+}\right)$são as aproximações por diferenças finitas regressivas e progressivas, respectivamente, da derivada de $\phi$ na direção $x$ (direção $y$ ).

O valor de $\Delta t$ é selecionado dentro do intervalo estabelecido pela condição Courant-FriedrichsLewy (CFL) [1]

$$
\Delta t \cdot \max \left\{\frac{|F| \cdot\left|\phi_{x}\right|}{|\vec{\nabla} \phi| \cdot \Delta x}+\frac{|F| \cdot\left|\phi_{y}\right|}{|\vec{\nabla} \phi| \cdot \Delta y}\right\}<1
$$

pela qual garante-se que a propagação dos dados numéricos dos contornos discretizados ocorra sob uma velocidade igual ou maior ao valor absoluto da velocidade $F$ de propagação dos dados físicos dos contornos reais, dada por $F=\phi^{0}-\phi_{\text {destino }}^{0}=\phi_{\text {origem }}^{0}-\phi_{\text {destino }}^{0}$, em ambas as direções $x$ e $y[8]$.

Pré-processadas as fatias origem e destino e delas extraídas as informações numéricas necessárias, executa-se o procedimento de metamorfose entre as fatias origem e destino. De posse do passo temporal $\Delta t$, a metamorfose é evoluída resolvendo a Equação 3.1 iterativamente, empregando-se o esquema de Roe-Fix [8] para o cálculo ponto a ponto da Hamiltoniana numérica $\hat{H}^{n}$.

O critério de parada da metamorfose fundamenta-se na métrica

$$
\mathrm{CP}=\left|\left\|\phi_{\text {origem }}^{n}-\phi_{\text {destino }}^{0}\right\|-\left\|\phi_{\text {origem }}^{n-1}-\phi_{\text {destino }}^{0}\right\|\right|
$$


na qual o símbolo $\|\cdot\|$ denota a norma-2 de seu argumento. Resultados práticos obtidos pelos autores confirmaram 0.25 como um parâmetro aplicável à maioria dos casos e que retorna as saídas (isto é, as fatias intermediárias) com a precisão numérica esperada.

Atingida a convergência, inicia-se o pós-processamento de todas as fatias (origem, destino e intermediárias geradas), etapa na qual os contornos são descentralizados e as fatias, redimensionadas às dimensões originais (se aplicável) - o pós-processamento, logo, executa as operações inversas às implementadas durante o pré-processamento.

Empilha-se o conjunto total de fatias, entre as originais e as intermediárias geradas pela metamorfose entre as duas de cada par de originais, em suas respectivas alturas, para formar a primeira saída visual do processo. Esse mesmo conjunto de fatias é passado como entrada ao procedimento de reconstrução, o qual irá gerar, por fim, a superfíce reconstruída.

\subsection{Particle Level Set}

A variante Particle Level Set segue a mesma sequência de passos supradescrita, diferenciando-se do original Level Set pela adição de três novos passos: sedimentação das partículas, atualização das partículas e correção do contorno via Particle Level Set.

A sedimentação é executada entre a determinação do passo temporal $\Delta t$ e a metamorfose entre as fatias origem e destino e ocorre sobre a primeira. Para cada vértice $(x, y)$ da grade de $\phi_{\text {origem }}^{0}$, testa-se se algum dos quatro vértices da célula à qual pertence o elemento em análise - a saber, além de $(x, y)$, os demais $(x+1, y),(x, y+1)$ e $(x+1, y+1)$ - encontra-se a uma distância máxima de três células do contorno. Em caso positivo, 8 partículas positivas e 8 negativas são depositadas no interior da célula contendo o elemento $(x, y)$ em seu canto superior esquerdo. Determinam-se as coordenadas e o sinal de cada partícula de maneira aleatória, respeitando os limites da célula para aquelas.

Conforme a padronização, as partículas negativas devem localizar-se na região interna ao contorno, na qual $\phi_{\text {origem }}^{0}<0$, e as positivas, na externa, em que $\phi_{\text {origem }}^{0}>0$. Sendo os sinais das partículas estabelecidos aleatoriamente, partículas tanto positivas quanto negativas encontramse, logo, em ambas as regiões ao final da deposição. Para adequar as posições das partículas conforme o padronizado, executa-se, na sequência, um procedimento de atração para a região apropriada da fatia, portanto.

Sejam, assim, as constantes $v_{\min }=0.1 \cdot \min \{\Delta x, \Delta y\}<v_{\max }=3 \cdot \max \{\Delta x, \Delta y\}$. Para cada partícula positiva, seleciona-se um valor aleatório $\phi_{\text {alvo }} \in\left[v_{\min }, v_{\max }\right]$; analogamente, para cada partícula negativa, $\phi_{a l v o} \in\left[-v_{\max },-v_{\min }\right]$. As partículas são, então, atraídas para a região correta segundo a equação de atração

$$
\vec{p}_{\text {novo }}=\vec{p}_{\text {original }}+\lambda \cdot\left[\phi_{\text {alvo }}-\phi_{\text {origem }}^{0}\left(\vec{p}_{\text {original }}\right)\right] \cdot \vec{N}\left(\vec{p}_{\text {original }}\right)
$$

com $\vec{p}_{\text {novo }}$ as novas coordenadas da partícula; $\vec{p}_{\text {original }}$ as originais; $\lambda$ um parâmetro de controle, inicialmente igual a $1 ; \phi_{\text {origem }}^{0}\left(\vec{p}_{\text {original }}\right)$ o valor interpolado de $\phi_{\text {origem }}^{0}$ para as coordenadas originais da partícula; e $\vec{N}\left(\vec{p}_{\text {original }}\right)$ o vetor unitário normal a $\phi_{\text {origem }}^{0}$ em $\vec{p}_{\text {original }}$. 
Resolve-se a Equação 3.2 iterativamente, para cada uma das partículas depositadas sobre a fatia. Se, ao fim da primeira iteração, alguma das coordenadas de $\vec{p}_{\text {novo }}$ não estiver dentro do domínio computacional da fatia, o parâmetro $\lambda$ é meado e a Equação 3.2, repetida para este novo $\lambda$, mantendo-se, porém, os valores originais de todas as outras variáveis.

Para as coordenadas $\vec{p}_{\text {novo }}$ retornadas pela reexecução da Equação 3.2, verifica-se se

$$
\phi_{\text {origem }}^{0}\left(\vec{p}_{\text {novo }}\right) \in\left[v_{\min }, v_{\text {max }}\right] \quad \text { ou } \phi_{\text {origem }}^{0}\left(\vec{p}_{\text {novo }}\right) \in\left[-v_{\max },-v_{\min }\right],
$$

conforme o sinal da partícula, positiva ou negativa, respectivamente. Em caso positivo, estabelece-se $\vec{p}=\vec{p}_{\text {novo }}$ como a nova posição da partícula; em caso negativo, a Equação 3.2, com o parâmetro $\lambda$ meado novamente e as outras variáveis igualmente mantidas, é executada pela terceira vez, a iteração corrente é encerrada e as coordenadas $\vec{p}_{\text {novo }}$ obtidas passam a desempenhar o papel de $\vec{p}_{\text {original }}$ na iteração seguinte, reatribuindo-se, inclusive, o valor 1 ao parâmetro $\lambda$.

Limitou-se em 10 o número de iterações da Equação 3.2 para cada partícula. Se, ao final da décima iteração, a partícula não se encontra na região apropriada, ela é descartada.

Depositadas e atraídas as partículas, o último procedimento relativo ao passo de sedimentação determina o raio $r_{p}$ de cada uma delas, segundo a convenção

$$
r_{p}= \begin{cases}r_{\max }, & \text { se } s_{p} \cdot \phi_{\text {origem }}^{0}(\vec{p})>r_{\max } \\ s_{p} \cdot \phi_{\text {origem }}^{0}(\vec{p}), & \text { se } r_{\min } \leq s_{p} \cdot \phi_{\text {origem }}^{0}(\vec{p}) \leq r_{\max } \\ r_{\min }, & \text { se } s_{p} \cdot \phi_{\text {origem }}^{0}(\vec{p})<r_{\min }\end{cases}
$$

na qual $r_{\min }=0.1 \cdot \min \{\Delta x, \Delta y\}, r_{\max }=0.5 \cdot \min \{\Delta x, \Delta y\}, s_{p}$ indica o sinal da partícula e $\phi_{\text {origem }}^{0}(\vec{p})$, o valor interpolado de $\phi_{\text {origem }}^{0}$ para a posição atraída $\vec{p}$ da partícula. Essa padronização assegura a tangência entre a borda da partícula e o contorno sempre que possível e, ainda, uma amostragem multiescala deste a partir do conjunto de partículas [6].

O passo seguinte de atualização das partículas desenvolve-se dentro do contexto da metamorfose entre as fatias origem e destino, imediatamente após a obtenção da matriz função distância sinalizada $\phi_{\text {origem }}^{n}$ e objetiva adequar a posição de cada uma delas à nova configuração da fatia origem. Cada partícula, logo, é evoluída de acordo com a EDP

$$
\frac{\vec{p}^{n}-\vec{p}^{n-1}}{\Delta t}=F\left(\vec{p}^{n-1}\right) \cdot \vec{N}\left(\vec{p}^{n-1}\right)
$$

sendo $\vec{p}^{n}$ as coordenadas da partícula após a $n$-ésima iteração da metamorfose a serem determinadas; $\vec{p}^{n-1}$, as coordenadas no início da $n$-ésima iteração; $\Delta t$, o passo temporal; $F\left(\vec{p}^{n-1}\right)$, o valor da velocidade $F$ interpolado para as coordenadas $\vec{p}^{n-1}$; e $\vec{N}\left(\vec{p}^{n-1}\right)$, o vetor unitário normal a $\phi_{\text {origem }}^{n-1}$ no ponto $\vec{p}^{n-1}$.

Na sequência, executa-se a correção do contorno via Particle Level Set, o último passo inerente à variante, por meio da identificação das partículas ditas escapadas, isto é, as quais, após a atualização de suas posições, localizam-se em regiões incorretas da fatia intermediária gerada e a uma distância do contorno estritamente maior do que a magnitude dos respectivos raios. Tais 
condições são testadas a partir do valor interpolado de $\phi_{\text {origem }}^{n}$ para as coordenadas $\vec{p}^{n}$ de cada uma das partículas sedimentadas e atualizadas.

Para cada partícula escapada, define-se a função Level Set local $\phi_{p}(\vec{v})$, enunciada como

$$
\phi_{p}(\vec{v})= \begin{cases}s_{p} \cdot r_{p}-\left|\vec{v}-\vec{p}^{n}\right|, & \text { se } \phi_{\text {origem }}^{n}(\vec{v}) \leq 0 \\ s_{p} \cdot r_{p}+\left|\vec{v}-\vec{p}^{n}\right|, & \text { se } \phi_{\text {origem }}^{n}(\vec{v})>0\end{cases}
$$

em que $s_{p}, r_{p}$ e $\vec{p}^{n}$ são, respectivamente, o sinal, o raio e as coordenadas da partícula escapada, e $\vec{v}$, as coordenadas de um vértice qualquer da grade da matriz $\phi_{\text {origem }}^{n}[10]$.

A Equação 3.4, calculada para cada um dos quatro vértices $\vec{v}$ da célula à qual pertence a partícula escapada, fornece os dados para a verificação de erros na matriz $\phi_{\text {origem }}^{n}$ : qualquer variação que haja entre $\phi_{p}(\vec{v})$ e o valor $\phi_{\text {origem }}^{n}(\vec{v})$ correspondente indica a possível existência de erros nos resultados numéricos presentes na matriz $\phi_{\text {origem }}^{n}$ [5].

A detecção e consequente correção dos erros numéricos principia-se com a declaração das matrizes auxiliares $\phi^{-}$e $\phi^{+}$, atribuindo a ambas a matriz $\phi_{\text {origem }}^{n}$ como valor inicial. Na ocasião de a partícula escapada ser positiva, calcula-se $\phi^{+}(\vec{v})=\max \left\{\phi^{+}(\vec{v}), \phi_{p}(\vec{v})\right\}$ para cada um dos quatro vértices $\vec{v}$ da célula à qual pertence a partícula; analogamente, para partículas escapadas negativas, determina-se $\phi^{-}(\vec{v})=\min \left\{\phi^{-}(\vec{v}), \phi_{p}(\vec{v})\right\}$.

Por meio da relação

$$
\phi_{\text {origem }}^{n}(x, y)= \begin{cases}\phi^{+}(x, y), & \text { se }\left|\phi^{+}(x, y)\right| \leq\left|\phi^{-}(x, y)\right| \\ \phi^{-}(x, y), & \text { se }\left|\phi^{+}(x, y)\right|>\left|\phi^{-}(x, y)\right|\end{cases}
$$

corrige-se, por fim, a matriz $\phi_{\text {origem }}^{n}$.

\section{RESULTADOS EXPERIMENTAIS}

A metodologia do processo de reconstrução foi implementada em ambiente MATLAB, por sua natureza matemática permitir uma codificação daquela, grandemente baseada em equações diferenciais parciais, com maior facilidade. Particularmente, para a etapa final de reconstrução, executou-se um script em linguagem Python, por este empregar o módulo de código aberto VTK e outras bibliotecas inerentes à linguagem. Para a verificação prática do processo como um todo, testes com dados sintéticos e testes com dados reais foram utilizados.

O teste Pirâmide, o primeiro com dados sintéticos, consistiu de sete fatias com contornos retangulares de dimensões decrescentes (Fig. 1). Conforme exposto anteriormente, a metamorfose via Level Set suaviza em demasia características finas das fronteiras em evolução, justificando a aplicação da variante Particle Level Set para a contenção, ou ao menos para o controle, dessa suavização. A configuração do teste Pirâmide alvejou, logo, analisar a capacidade real do Particle Level Set para a satisfação desse objetivo proposto.

A Figura 2 apresenta a fatia intermediária gerada, tanto pela metamorfose via Level Set quanto pela via Particle Level Set, ao final da terceira iteração do procedimento. Um exame minucioso 


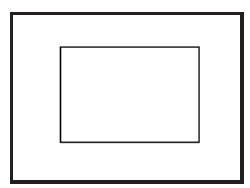

(a) Fatia 1

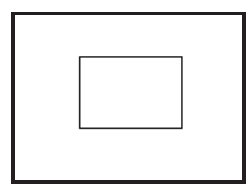

(b) Fatia 2

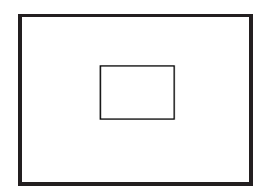

(c) Fatia 3

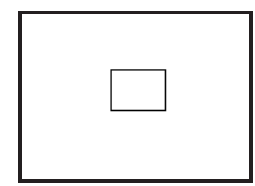

(d) Fatia 4

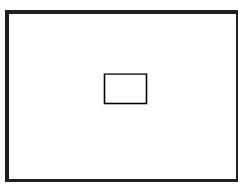

(e) Fatia 5

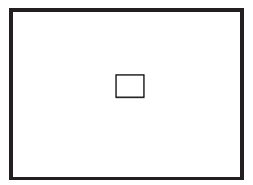

(f) Fatia 6

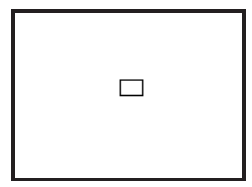

(g) Fatia 7

Figura 1: Fatias originais - Pirâmide.

da figura em questão revela a sutileza do Particle Level Set na preservação dos cantos do contorno retangular sob deformação, corroborando, portanto, o controle da suavização hipotetizado.

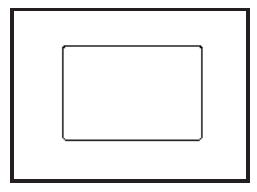

(a) Level Set

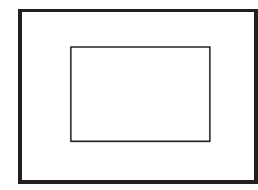

(b) Particle Level Set

Figura 2: Terceira fatia intermediária gerada - Pirâmide.

O segundo teste com dados sintéticos, Castiçal, objetivou investigar a adaptabilidade da metodologia proposta para o tratamento de fatias com mudanças topológicas acentuadas, isto é, contornos dividindo-se em dois ou mais outros distintos ou, inversamente, unindo-se em um único (Fig. 3).

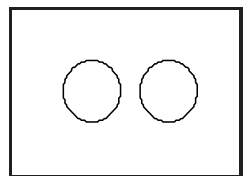

(a) Fatia 1

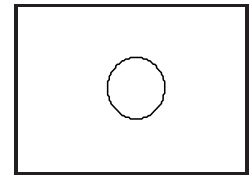

(b) Fatia 2

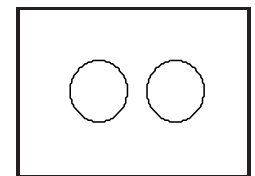

(c) Fatia 3

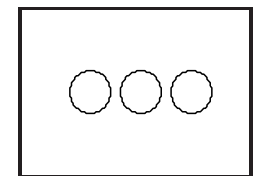

(d) Fatia 4

Figura 3: Fatias originais - Castiçal.

As superfícies reconstruídas para os casos Pirâmide e Castiçal, a partir do conjunto de fatias originais tão somente e do total de fatias, entre originais e intermediárias geradas, são apresentadas nas Figuras 4 e 5, respectivamente. Uma análise comparativa entre as duas superfícies de cada teste atesta a evolução da qualidade da superfície resultante; entretanto, conforme ilustrado na Figura 4, especificamente, pela metamorfose via Particle Level Set, os contornos foram intensamente deformados no início da metamorfose e praticamente inalterados ao final, o que originou uma pilha de fatias intermediárias com um notável grau de semelhança entre si, à conclusão 
da metamorfose, e, por consequência, uma superfície reconstruída possivelmente divergente da esperada teoricamente, mais suave.

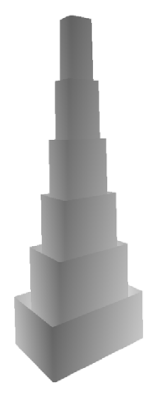

(a) Somente fatias originais

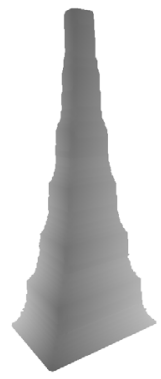

(b) Particle Level Set

Figura 4: Superfícies reconstruídas - Pirâmide.

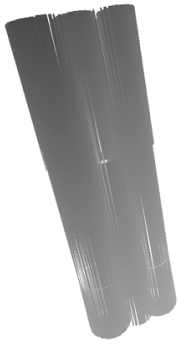

(a) Somente fatias originais

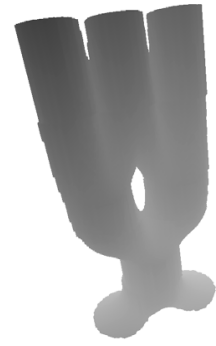

(b) Level Set

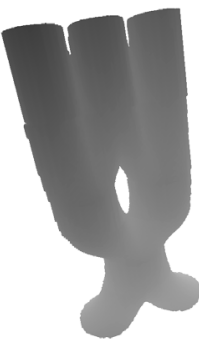

(c) Particle Level Set

Figura 5: Superfícies reconstruídas - Castiçal.

Particularmente ao caso Castiçal, nota-se, com maior veemência, a habilidade de ambos os métodos Level Set e Particle Level Set para a reconstrução de superfícies a partir de fatias planas paralelas, quando a amostragem destas evidencia-se defectiva de alguma forma: com o emprego da metodologia neles baseada, foi possível delinear uma superfície originalmente amorfa em sua forma apropriada. Além disso, uma análise mais criteriosa das Figuras 5(b) e 5(c) constata a capacidade da variante em controlar a velocidade da metamorfose, o que possibilitou o desenho da base do objeto com uma maior verossimilhança, atestando o aprimoramento qualitativo proporcionado pela reconstrução por meio da metamorfose via Particle Level Set.

Para os testes com dados reais, foram reconstruídos dois ossos do corpo humano, Clavícula e Ilíaco. Esses dados, disponíveis em [3], foram adquiridos por Tomografia Computadorizada no formato DICOM (Digital Imaging and Communications in Medicine). De modo a ilustrar com maior eloquência a capacidade afirmada de a metodologia proposta gerar os dados complementares necessários, para estes testes, as Figuras 6 e 7 exibem as correspondentes representações ósseas reconstruídas por meio do empilhamento do conjunto completo de fatias, entre originais e intermediárias geradas, ao invés da renderização das superfícies. 


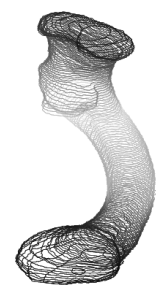

(a) Somente fatias originais

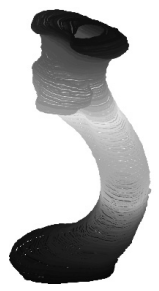

(b) Particle Level Set

Figura 6: Fatias empilhadas - Clavícula.

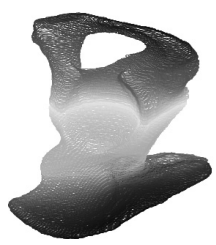

(a) Somente fatias originais

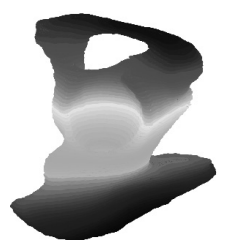

(b) Particle Level Set

Figura 7: Fatias empilhadas - Ilíaco.

Tabela 1: Métricas dos conjuntos de fatias.

\begin{tabular}{|l|c|c|c|c|}
\hline \multirow{2}{*}{ Métricas } & \multicolumn{4}{c|}{ Conjuntos de fatias } \\
\cline { 2 - 5 } & Pirâmide & Castiçal & Clavícula & Ilíaco \\
\hline Número de fatias & 7 & 4 & 141 & 253 \\
\hline Dimensões (A× L) das fatias & $70 \times 100$ & $70 \times 100$ & $110 \times 110$ & $144 \times 128$ \\
\hline Distância vertical entre as fatias & uniforme: 40 & $50-50-100$ & uniforme: 1 & uniforme: 0.5 \\
\hline Número de pixels por fatia & & & & \\
Mínimo & 44 & 306 & 21 & 111 \\
Máximo & 278 & 648 & 168 & 819 \\
Média no conjunto & 134.29 & 544.50 & 115.52 & 567.69 \\
\hline
\end{tabular}

A Tabela 1 detalha as métricas relevantes dos conjuntos de fatias de cada caso de teste trabalhado. Como citado, os testes com dados sintéticos foram construídos de modo a avaliar o comportamento do processo proposto frente a casos cuja amostragem de fatias revela-se falha de alguma forma; por sua vez, os testes Clavícula e Ilíaco são mais próximos ao mundo real, apresentando uma amostragem mais fina das fatias.

A Tabela 2 minucia as estatísticas quantitativa e qualitativa do procedimento de geração das fatias intermediárias, pormenorizadas para os quatro casos de testes apresentados. Analisando os dados expostos, nota-se o alto custo da metamorfose via Particle Level Set, comparativamente à metodologia via Level Set, exigido para o aperfeiçoamento praticado pelo primeiro método, custo esse grandemente dependente das características das fatias originais, como, por exemplo, 
Tabela 2: Estatísticas dos testes.

\begin{tabular}{|c|c|c|c|}
\hline \multirow{2}{*}{ Teste } & \multirow{2}{*}{ Estatísticas } & \multicolumn{2}{|c|}{ Método } \\
\hline & & LS & PLS \\
\hline \multirow{2}{*}{ Pirâmide } & Total de fatias intermediárias geradas & 223 & 250 \\
\hline & Tempo médio $(s)$ de geração de cada fatia intermediária & 1.60 & 20.68 \\
\hline \multirow{2}{*}{ Castiçal } & Total de fatias intermediárias geradas & 751 & 504 \\
\hline & Tempo médio $(s)$ de geração de cada fatia intermediária & 1.20 & 23.27 \\
\hline \multirow{2}{*}{ Clavícula } & Total de fatias intermediárias geradas & 1544 & 1578 \\
\hline & Tempo médio $(s)$ de geração de cada fatia intermediária & 1.56 & 20.48 \\
\hline \multirow{2}{*}{ Ilíaco } & Total de fatias intermediárias geradas & 2448 & 2460 \\
\hline & Tempo médio $(s)$ de geração de cada fatia intermediária & 2.35 & 33.84 \\
\hline
\end{tabular}

quantidade de pixels e topologia dos contornos nelas presentes; ademais, observa-se que ambos os métodos, no geral, geram, aproximadamente, quantidades iguais de fatias intermediárias.

\section{CONCLUSÕES E TRABALHOS FUTUROS}

O trabalho objetivou analisar a viabilidade do Level Set e de sua variante Particle Level Set para a reconstrução tridimensional. Com base nos resultados experimentais, pode-se confirmar a habilidade de ambos os métodos para o objetivo proposto, sendo possível expandir o potencial do Particle Level Set para o controle da suavização inerente ao Level Set, por meio de aperfeiçoamentos teóricos e computacionais na metodologia descrita.

Como uma solução para a questão da velocidade não uniforme da metamorfose observada no teste Pirâmide (Fig. 4), Nilsson et al. [7] propõem um esquema no qual, em sua essência, as metamorfoses inerentes a todos os pares são executadas ao mesmo tempo, reformulando a velocidade $F$ em uma função contínua e uniforme.

Para potencializar os resultados retornados pelo Particle Level Set, pode-se ajustar a quantidade de partículas sedimentadas, por célula da grade, de acordo com o nível de resolução almejado, sendo essas duas variáveis diretamente proporcionais. Contudo, ponderação mostra-se necessária na definição da primeira, pois um aumento desmesurado em seu número afeta negativa e profundamente a duração do processo.

Neste ponto, uma possibilidade para a aceleração do processo reside no método Narrow Band Level Set [9], uma adaptação mais eficiente do original, em termos de tempo de execução, pelo qual apenas os pontos da grade pertencentes ao interior de uma banda estreita de largura $k$, posicionada ao redor da fronteira dinâmica, são atualizados. Uma abordagem mais baixo nível reside nas ferramentas disponibilizadas pelo MATLAB que acionam e otimizam a computação em paralelo, como a capacidade de expansão do paralelismo para uma rede de computadores, inclusive com o uso da computação em nuvem, ou o aproveitamento da potência da unidade de processamento gráfico (GPU). 
Fundamentando-se no apresentado, conclui-se sobre a viabilidade tanto do método Level Set quanto da variante Particle Level Set para a reconstrução de superfícies a partir de fatias planas paralelas: enquanto o primeiro destaca-se eficiente para casos generalizados, recomenda-se o emprego do segundo a situações em que a preservação de características finas dos contornos e da superfície a ser reconstruída é imprescindível e impera sobre o tempo de execução do processo, para, por exemplo, a avaliação mais precisa de informações quantitativas do objeto em estudo, tais como a área lateral e o volume.

\title{
AGRADECIMENTOS
}

Os autores são gratos à FAPESP, ao CNPq e à CAPES pelo apoio financeiro.

\begin{abstract}
Evolving interfaces were initially focused on solutions to scientific problems in Fluid Dynamics. With the advent of the more robust modeling provided by Level Set method, their original boundaries of applicability were extended. Specifically to the Geometric Modeling area, works published until then, relating Level Set to tridimensional surface reconstruction, centered themselves on reconstruction from a data cloud dispersed in space; the approach based on parallel planar slices transversal to the object to be reconstructed is still incipient. Based on this fact, the present work proposes to analyse the feasibility of Level Set to tridimensional reconstruction, offering a methodology that simultaneously integrates the proved efficient ideas already published about such approximation and the proposals to process the inherent limitations of the method not satisfactorily treated yet, in particular the excessive smoothing of fine characteristics of contours evolving under Level Set. In relation to this, the application of the variant Particle Level Set is suggested as a solution, for its intrinsic proved capability to preserve mass of dynamic fronts. At the end, synthetic and real data sets are used to evaluate the presented tridimensional surface reconstruction methodology qualitatively.
\end{abstract}

Keywords: Level Set, Particle Level Set, metamorphosis, reconstruction.

\section{REFERÊNCIAS}

[1] R. Courant, K. Friedrichs \& H. Lewy. On the Partial Difference Equations of Mathematical Physics. Journal of Research and Development, 11 (1967), 215-234.

[2] C.A.Z. Barcelos. Restauração e Análise de Imagens via Equações Diferenciais Parciais. Revista Tendências em Matemática Aplicada e Computacional, 3(2) (2013), 1-13.

[3] S. Barré. http://www.barre.nom.fr/medical/dicom2/, (2013).

[4] D. Breen \& R. Whitaker. A Level-Set Approach for the Metamorphosis of Solid Models. IEEE Transactions on Visualization and Computer Graphics, 7(2) (2001), 173-192.

[5] D. Enright. "Use of the Particle Level Set Method for Enhanced Resolution of Free Surface Flows", Tese de Doutorado, Stanford University, Stanford, California, Estados Unidos (2002). 
[6] D. Enright, F. Losasso \& R. Fedkiw. A Fast and Accurate Semi-Lagrangian Particle Level Set Method. Computers and Structures, 83(6-7) (2005), 479-490.

[7] O. Nilsson, D. Breen \& K. Museth. Surface Reconstruction via Contour Metamorphosis: An Eulerian Approach with Lagrangian Particle Tracking, em "Proceedings of the 16th IEEE Visualization", pp. 407-414, Washington DC, Estados Unidos (2005).

[8] S. Osher \& R. Fedkiw. "Level Set Methods and Dynamic Implicit Surfaces”, Springer-Verlag, New York (2002).

[9] J.A. Sethian. "Level Set Methods and Fast Marching Methods: Evolving Interfaces in Computational Geometry, Fluid Mechanics, Computer Vision, and Materials Science", Cambridge University Press, New York (1996).

[10] Z. Wang, J. Yang \& F. Stern. An Improved Particle Correction Procedure for the Particle Level Set Method, Journal of Computational Physics, 228(16) (2009), 5819-5837.

[11] Y. Wang, L. Wang, S. Xiang \& C. Pan. Level Set Evolution with Locally Linear Classification for Image Segmentation, "Proceedings of the 18th IEEE International Conference on Image Processing", Bruxelas, Bélgica, (2011), 3361-3361.

[12] S.Y. Yeo, X. Xie, I. Sazonov \& P. Nithiarasu. Level Set Segmentation with Robust Image Gradient Energy and Statistical Shape Prior, "Proceedings of the 18th IEEE International Conference on Image Processing”, Bruxelas, Bélgica, (2011), 3397-3400. 